\title{
Aspects of Energy Transformation and Energy Control Capabilities in Electric Machines with a Liquid-Metal Working Body
}

\author{
Aleksiy A. Maksimov, Maksim Y. Khatsayuk, Viktor N. Timofeev \\ Siberian Federal University, Krasnoyarsk, Russia
}

\begin{abstract}
The article contains mathematical simulation of the aluminum melt mixing in the alloying furnace bath exposed to the travelling electromagnetic field of MHD stirrer. Based on the designed mathematical model, there has been assessed the impact of the normal component of electromagnetic forces in the process of MHD-stirring the liquid aluminium. The analysis has been completed on the computational simulation results at different ratio adopted for the tangential and normal components of the electromagnetic force and the have been proposed recommendations for effective stirring of aluminum melts.
\end{abstract}

Keywords - magnetohydrodynamics, computational model, MHD stirrer, metallurgy, electromagnetic forces.

\section{INTRODUCTION}

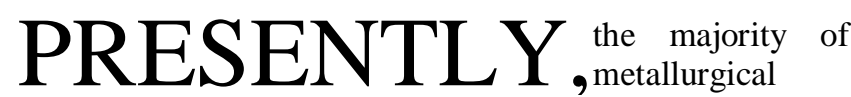
smelters employ magnetohydrodynamic (MHD) stirrers to mix aluminum alloys in reverberatory and alloying furnaces. The use of such stirrers enables to eliminate the mechanical impact on the melt, to increase production output of melting furnaces and ensures uniformity of the melt in its temperature and chemical composition at the expense of heat and mass transfer [1-3].

The MHD stirring units are designed on the basis of single-sided linear induction machines affecting the bath with the melt. One of their features is the presence of normal (repulsive) component of electromagnetic forces (Fig. 1). The distribution of electromagnetic forces and the predominance of one or the other spatial component determines the future character of the melt circulation in the closed baths of reverberatory and alloying furnaces. Of greatest interest is the effect of pulling component in relation to the general vector of force.

Thus, in order to analyze the effectiveness of the impact produced by the normal component on melt stirring process, there has been completed the computational simulation over the hydrodynamic processes with various normal components of electromagnetic forces in relation to the tangential component.

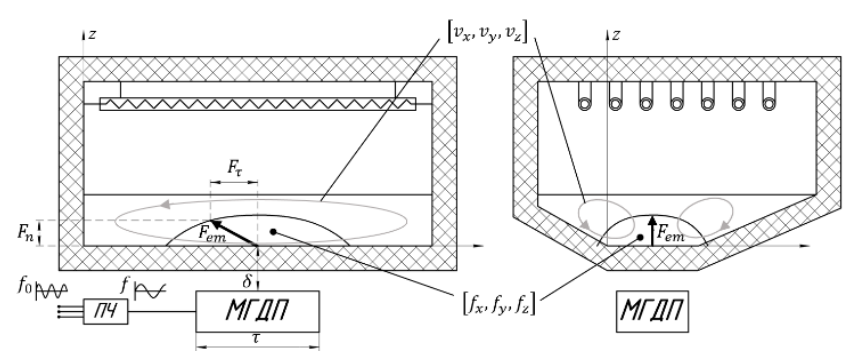

Fig. 1. Sketch of the system "Furnace - MHD-stirrer".

\section{PROBLEM STATEMENT}

The description of the mathematical model of the system "MHD-stirrer-melt" have been well reviewed in article [4], which served as the basis for stirring simulation (Fig. 2). During the transfer of the distribution matrix for the electromagnetic forces in the hydrodynamic part of the task, the normal component was artificially modified.

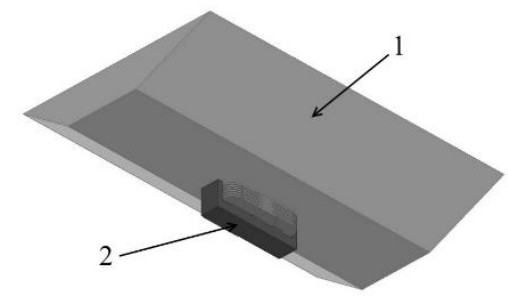

Fig. 2. General view of the system of "melt-MHD-stirrer": 1 - melt; 2 MHD stirrer

LES (Large Eddy Simulation) was chosen as the turbulence model for hydrodynamics. In the "furnaceMHD-stirrer" system the electromagnetic and hydrodynamic processes were resolved in the threedimensional unsteady setting through the conjugation of ANSYS Classic and ANSYS CFX programs.

\section{THEORY}

The computational calculation of the electromagnetic task resulted in the spatial distribution of vector components of volume electromagnetic forces in the melt:

$$
\left[f_{x}, f_{y}, f_{z}\right] \text {, }
$$


where $f_{x}$ - longitudinal (pulling), $f_{y}$ - latitudinal, and $f_{z}$ - normal (pushing) component. Component-wise vector integration allows to identify the cumulative electromagnetic force most fit for the analysis. In actual furnaces, the longitudinal dimension of the bath is more than the latitudinal dimension of the inductor, thus, the resulting integral transverse force is balanced:

$$
F_{y}=\int f_{y} d v=0 \text {. }
$$

Therefore, the resulting force vector is as follows:

$$
\left[\int f_{x} d v, 0, \int f_{z} d v\right]=\left[F_{\tau}, 0, F_{n}\right]
$$

with a relative pulling force

$$
F_{\tau}^{\prime}=F_{\tau} / \sqrt{F_{n}^{2}+F_{\tau}^{2}} \text {. }
$$

The existing analytical methods for the calculation of unilateral linear induction machines [5] enable to identify the main factors impacting the value $F_{\tau}{ }^{\prime}$ :

1. Quality factor $\varepsilon=\mu_{0} \omega \gamma s(\tau / \pi)^{2}$, where $\omega=2 \pi f$ - circular frequency, $f$ - frequency, $s$-sliding, $\gamma$ - electrical conductivity, $\tau$ - polar pitch.

2. Relative size of the gap $\delta / \tau$.

3. The design of the inductor windings (number of $M$ phase zones, winding type, presence of compensating elements, etc.) [5].

An example of the nature of the impact produced by these factors on the value $F_{\tau}{ }^{\prime}$, for some specific cases without taking into account the transverse effect is presented in Fig. 3.
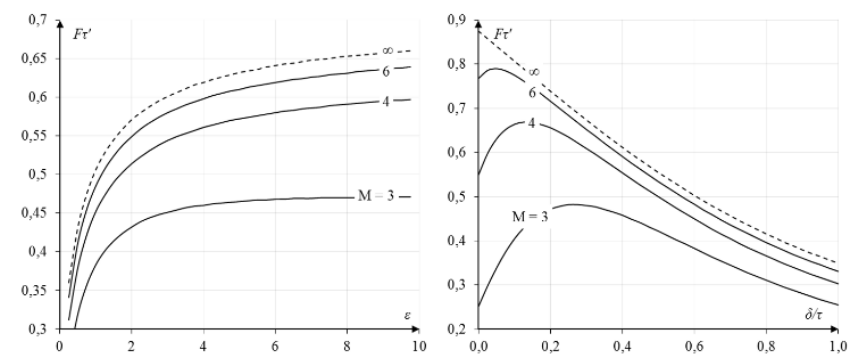

Fig. 3 - Evaluation of the impact of quality and relative gap for linear induction machines with different number of phase zones on the value of the pulling force.

As seen from the presented relations, the nature (inclination) of the resulting electromagnetic forces vector, and, therefore, its volumetric distribution varies within a wide range and is highly dependent on the configuration and parameters of the linear induction machine. In its turn, there is the question open regards the influence produced by the nature of these forces distribution on the emerging field of velocities within a confined volume of the melt.

As a result, the basic distribution of electromagnetic forces in the hydrodynamic task is converted as follows: $\left[f_{x}, f_{y}, f_{z}\right] \rightarrow\left[f_{x}, f_{y}, f_{z}\left(1-F_{\tau}{ }^{\prime}\right)\right]$.

The basic value $F_{\tau}{ }^{\prime}=0,43$ corresponds to the standard settings of the MHD-stirrer inductor in the start mode. To assess the impact produced by a normal component of electromagnetic forces on the melt, a number of values $F_{\tau}{ }^{\prime}$ from the base unit were taken into account. Fig. 4 shows how the resulting vector of electromagnetic force and the field of volumetric forces corresponding to its extreme positions are changed in the longitudinal section.

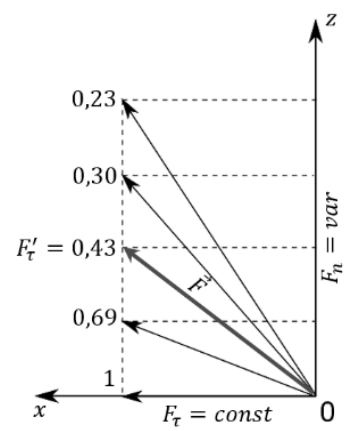

a)

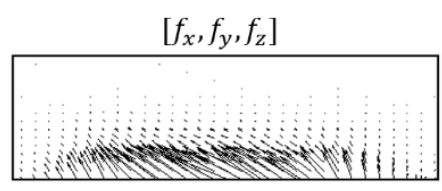

$\left[f_{x}, f_{y}, f_{z} \cdot\left(1-F_{\tau}^{\prime}\right)\right]$

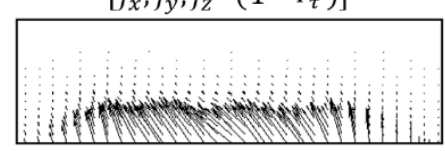

b)
Fig. 4. The electromagnetic forces vector and its projection at different $F_{\tau}{ }^{\prime}$ (a) and corresponding to two extreme positions of the volumetric forces field

Further, the results of the studies completed on the the impact of the changes $F_{\tau}{ }^{\prime}$ made on the hydrodynamic processes on the example of the actual bath in the industrial metallurgical furnace with the capacity of 70 tons with MHD-stirring (number of phases $M=4$, the type of winding: intersecting, two-phase). Since the experiments under conditions of the existing production and physical simulation of MHD processes face considerable difficulties, the studies were carried out through the application of theoretical methods. The description and configuration of the hydrodynamic model and turbulence models are given in the previous work [6].

\section{ANALYSIS OF THE RESULTS}

Based on the model described, there has been performed the calculation and main hydrodynamic characteristics for the set task have been obtained. Fig. 5 shows the distribution of velocity in longitudinal section and in the volume of the bath furnace for the basic calculation point and two extreme $\left(F_{\tau}{ }^{\prime}=M A X ; B A S E ; M I N=1,00 ; 0,43 ; 0,23\right)$

As it can be seen from Fig. 5, in the conditions of the normal component in the electromagnetic force, the main stream of the liquid metal is closed at a more elongated trajectory with the maximum value of velocity near the bath bottom. As the normal component increases, the nature of the flow becomes more local in nature with the increase of mass transfer in a vertical direction. 

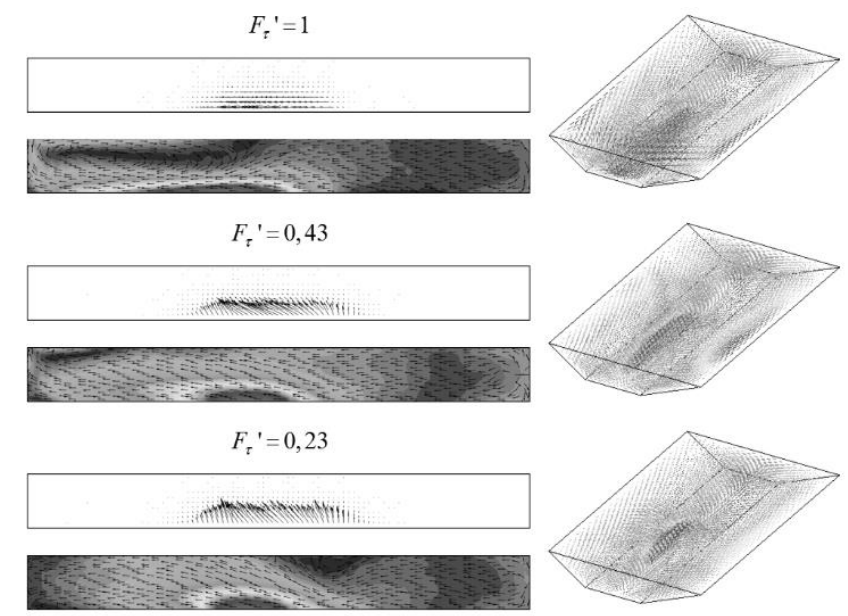

Fig. 5. Distribution of the velocity vector with the relevant applied electromagnetic forces

To quantitatively assess the patterns of flow parameters change and modes for the inductor operation modes, there has been analyzed the maximum velocity in the volume of a bath and total kinetic energy (Fig. 6). Kinetic energy characterizes the intensity of mixing, while the maximum velocity in the area of the inductor allows to evaluate the inductor's operation mode (sliding). The relations are complex, however, there is noticed the trend to increase these parameters while reducing the influence of the normal component. Since the kinetic energy is related to the average velocity in the melt, its character should have been repeating the diagram of the gained velocity. However, there is no such relation detected and we can assume that for each respective level of melt in the bath and bath geometry in general there exist its own optimal point of relative component of a pulling force.

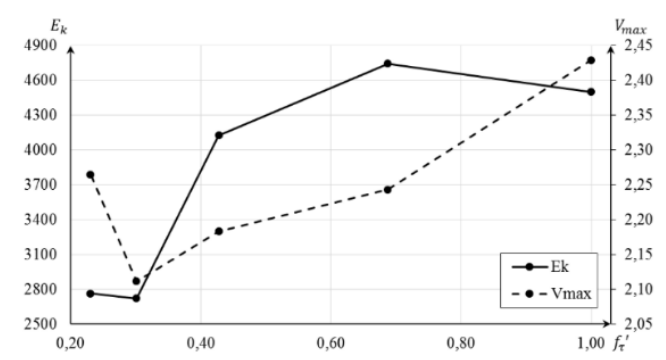

Fig. 6. The diagram of a maximum velocity and kinetic energy relation to the normal component magnification ratio

To study the process of stirring the melt the task was added with the model of mass concentration with a neutral buoyancy (markers). The initial conditions for the markers distribution correspond to the characteristic points of loading the alloying components of the melt during operation of furnaces at the production site. The dynamics of dissolving markers in the conditions of MHD-stirring for the base calculation point and two extreme ones is presented in Fig. 7.

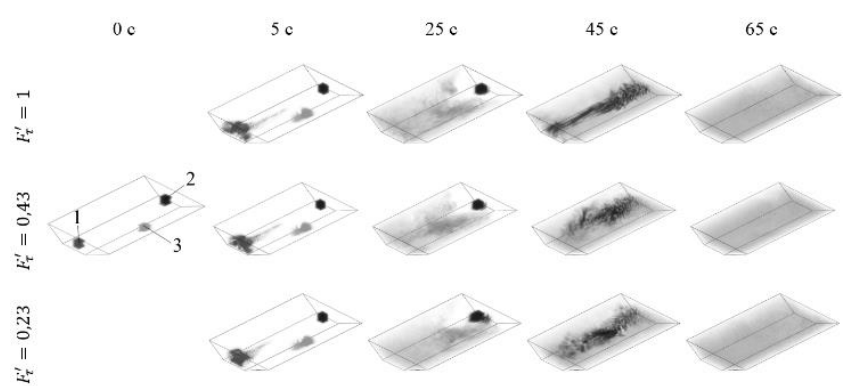

Fig. 7. Dynamics of the markers dissolution in the bath

For the ease of the analysis, the results of the markers concentration levelling (stirring) depending on the time in the bath are presented in the form of diagrams (Fig. 8). Yaxis shows the percentage of marker dissolution in the volume of the bath.
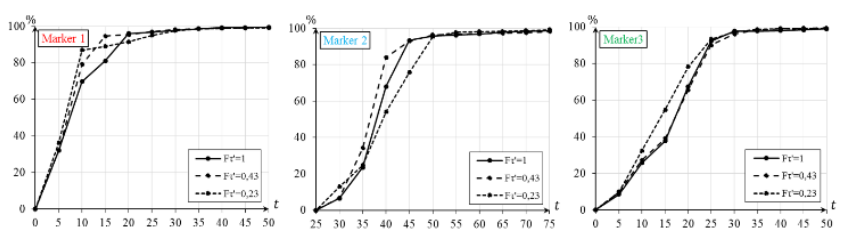

Fig. 8. Levelling markers concentration in the volume of the bath at different values $F_{\tau}$

According to the results of concentration task solution presented above, although there was a significant change in the nature of the melt circulation, if $F_{\tau}{ }^{\prime}$ is changed, no significant and regular change in the speed of leveling is observed. It is clear, however, that at a certain change in geometry of the furnace bath or melt level, the differences may become more vivid.

Below are the results of the calculation under the same conditions for the bath increased up to 140 tons at the expense of its lengthening. The resulting vector field of speeds is shown in Fig. 9.

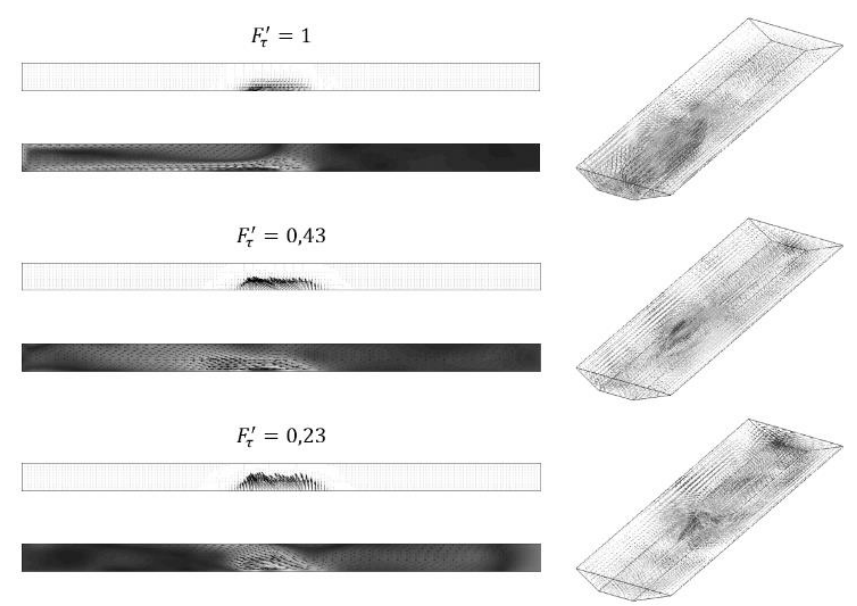

Fig. 9. Distribution of the velocity vector with the relevant applied electromagnetic forces for the lengthened bath.

The diagrams of the concentration change dynamics (Fig. 10) express the impact of the normal component. Marker 1 located in front of the inductor is quicker in levelling in the absence of a normal component. The increase in the normal component reduces the rate of stirring. The dynamics of 
stirring the second marker has the opposite effect, since the marker is located behind the inductor. The acceleration of its stirring at the low pulling force can be explained by the increase in the flow turbulent component and generation of the travelling whirl. To resolve this problem at the production site, the inductors normally operate in the mode of periodic reversing. The third marker repeats in its nature the previous result as it is located next to the inductor.
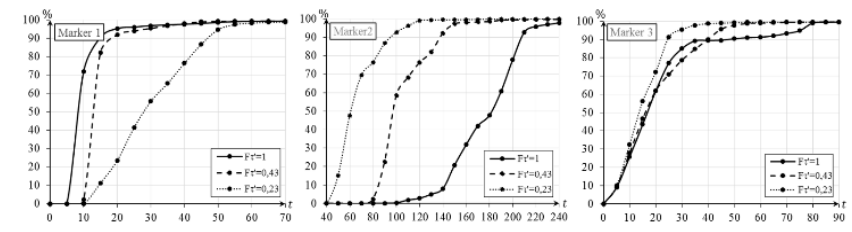

Fig. 10. Distribution of markers in the bath volumes at different values $F_{\tau}{ }^{\prime}$ for the increased bath.

\section{FINDINGS AND CONCLUSIONS}

The article assessed the impact of the electromagnetic forces field distribution, namely application of a normal component in the process of formation of hydrodynamic MHD-flows in a bath with liquid metal. The results obtained enable to make several major conclusions about its impact:

1. When the relative value of the normal component is over $30 \%$ in the resulting force vector, there is detected the reduction in the number of movements in the furnace bath. The electromechanical operation mode of the inductor changes slightly.

2. The reduction of a normal component leads to more aimed, orderly and predictable nature of the melt circulation. However, when developing the technology of alloy preparation it is required to take into account the geometry of the melt (working level of melt, furnace length, cross-sectional shape of a bath) in the bath in each specific case.

3. The compensation of the longitudinal edge effect produced by the single-sided linear induction machine when working in a closed melt enables to expand the scope of intense currents in the direction of its actions (in this example, in the horizontal plane). It is worth noting that in this case the inductor must be located at the edge of the furnace or there should be applied a periodic reversing mode.

These results are recommendations for the design of linear induction machines with a liquid metal working body and their control systems to achieve optimal technological regimes.

\section{ACKNOWLEDGEMENTS}

This research has been completed with the financial support of the Russian Fund of Fundamental Research, the Government of Krasnoyarsk Territory, Krasnoyarsk Territory Fund of Science within the framework of Research Project No. 18-48-242013 "Research into the impact of spatial and time-and-frequency distributions of electromagnetic forces in the melt on the nature of the MHD processes in liquid metal" and "Research into dynamic characteristics of turbulent flows of melt under electromagnetic crystallization and its impact on the structure and properties of continuously cast small section billets made of new aluminum alloys for the production of thin aerospace wire".

\section{REFERENCES}

[1] Continuous casting of aluminum alloys: Reference book/V.I. Napalkov, T.V. Cherepok, S.V. Makhov, Y.M Chernovol-Moscow: Intermet Engineering, 2005. - 512 p.

[2] Vertel L.A. Magnetichydrodynamics in metallurgy. -Moscow: Metallurgy, 1975. $-287 \mathrm{p}$

[3] Application of MHD units in metallurgy: student book for independent work / edited by V.N. Timofeev, E.A. Golovenko, E.V. Kuznetsov - Krasnoyarsk: Siberian Federal University, 2007, 192 p.

[4] Theoretical design baselines for MHD-stirrers of molten metals, Magnetohydrodynamics Vol.52, 2016, No. 4, pp. 495-506

[5] Voldek A.I. Inductive magnetic hydrodynamic machine with liquidmetal working body. Leningrad: Energiya, 1970. 272 p.

[6] Maksimov A. A., Khatsayuk M. Y., Timofeev V. N. Comparative evaluation of the results of numerical simulation of hydrodynamic processes in the system "bath with melts-MHD-stirrer". The magazine of the Siberian Federal University. Technics and Technology, 2018, 11 (2), 138-147 pp.

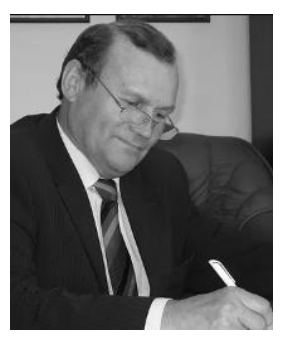

Viktor N. Timofeev was born in 1950, in the Ermolayevo Village, Balakhta District, Krasnoyarsk Territory. In 1974, he completed his education in Krasnoyarsk Polytechnical Institute majoring in Power Plants. In 1978, he was awarded the degree of the Candidate of Technical Sciences. In 1994, he was awarded the degree of the Doctor of Technical Sciences.

Area of expertise: electromagnetic fields analysis; research into electrical process equipment and MHD technology for metallurgical purposes; linear electric machines; methods of complex mathematical and physical simulation; computer aided design of electrical process equipment.

He is the member of the Thesis Board for awarding titles of Candidate and Doctoral degree, the author of over 200 scientific publications, including 2 monographs, 8 educational books, and 70 inventor's certificates and patents. Since 1990, he has been heading the "Research and Production Center of Magnetic Hydrodynamics", Ltd ("RPC Magnetic Hydrodynamics" Ltd), which designs and supplies electrical process equipment and technology to smelters.

$\mathrm{He}$ is Doctor of Technical Sciences, Professor, Academician in Academy of Electrotechnical Sciences, Head of the Department of Electrical Engineering and Electric Process Equipment in the Polytechnical Institute of the Siberian Federal University, member of the International Scientific Committee of the continuous international conference :Topical Problems of Theory and Practice of the Induction Heating", has been employed with the Polytechnical Institute of the Siberian Federal University (formerly Krasnoyarsk State Technical University) since 1978, member of the Expert Council in Association of Engineering Education of Russia. In 2002, he was awarded the diploma of the winner of the all-Russian competition "Engineer of the year" in the nomination "Electrical equipment". In 2006, he was awarded a label pin of "Honorary worker of science and technology of the Russian Federation".

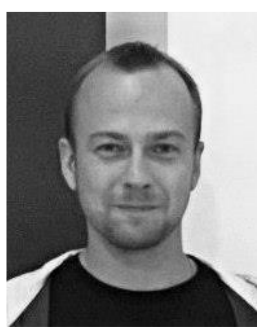

Maksim Y. Khatsayuk was born in 1988 in Minusinsk Town, Krasnoyarsk Territory. He was qualified as an Engineer in specialty of "Electric process units and systems" in the 
Siberian Federal University (Krasnoyarsk) in 2010, and was awarded the degree of Candidate of Technical Sciences specializing in "Electric machines" in the Ural Federal University (Yekaterinburg) in 2014.

In 2014 - 2018, he held the position of Teaching Assistant, Senior Lecturer, Associate Professor of the Electrical Equipment and Electrical Process Technology Department in the Polytechnic Institute of the Siberian Federal University. In 2010 - 2018, he is an Engineer, Researching Engineer, Head of the Mathematical Simulation Department at "RPC Magnetic Hydrodynamics" Ltd. He is the author of 4 methodological and educational books, 65 publications, 12 patents in the field of electrical process technology and MHD-equipment for metallurgy, acted as a Manager and Executor of $14 \mathrm{R} \& \mathrm{D}$ projects. Area of expertise: research into related electromagnetic, thermal, magnetic and hydrodynamic processes in metallurgical units with a view to obtain materials of new physical-mechanical properties and enhanced safety, environment and energy efficiency of the electrical process equipment.

Prize winner of the contest held by the Krasnoyarsk City Mayor, the State Award of the Krasnoyarsk Territory, Award of the Strategic Research Fund "Siberian Club", Award of the IFC Bank in scientific nominations, medal of All-Russian Exhibition of Scientific and Technica Creativity of Youth "For achievements in the scientific and technical creativity".

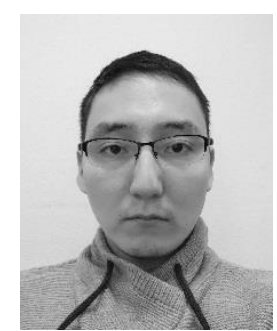

Aleksiy A. Maksimov was born in 1992, in the Lensk town, Republic of Sakha (Yakutia). In 2014, he was awarded the Bachelor's Degree in Electric Engineering, Mechanics, and Electrotechnics. In 2016, he completed the Master's Degree program in Electrotechnics in Metallurgy. Aleksiy Maksimov is presently taking his post-graduation studies and is as an Assistant at the Department of "Electrotechnics and Electric Engineering" in Siberian Federal University. He is the author of more than 15 articles. Area of expertise: conjugate electromagnetic, thermal and hydrodynamic calculations, MHD technology. 